\title{
Implications of noise on the reference signal in feedforward control of harmonic disturbances
}

\author{
Robert L. Clark \\ Department of Mechanical Engineering and Materials Science, Duke University, Durham, North Carolina \\ 27708-0300 \\ Gary P. Gibbs \\ Department of Mechanical Engineering, Virginia Polytechnic Institute and State University, Blacksburg, \\ Virginia 24061
}

(Received 30 March 1994; accepted for publication 22 June 1994)

\begin{abstract}
The purpose of this Letter is to detail the effect of random (zero-mean) noise on the reference input of an adaptive feedforward filter in the control of single-frequency disturbance inputs. Results from this study indicate that if system identification is required to execute the adaptive algorithm (as is the case with the filtered-x LMS algorithm), then the sample rate should be set at 4 samples per period to minimize the number of finite impulse response filter coefficients needed to compute an accurate estimate of the filtered reference signal for multi-channel control. However, results from this study demonstrate that the sample rate has very little effect on the minimum mean-squared error that can be achieved with the adaptive control filter, owing to the reverberant characteristics of the dynamics system.
\end{abstract}

PACS numbers: $43.40 . \mathrm{Vn}$

\section{INTRODUCTION}

Active feedforward control has been the subject of considerable research and implementation in recent years as a result of a widely referenced paper by Elliot et al. (1987) whereby the authors developed a multiple-input/multipleoutput version of the filtered-x LMS algorithm (Widrow and Stearns, 1985) for control of persistent disturbances. Since this publication, adaptive feedforward control has been the subject of a number of applications and investigations. The purpose of this letter is to detail practical concerns in feedforward control of harmonic signals with respect to random noise on the reference signal and investigate the effect that the sampling rate has on the performance characteristics.

The work presented is applicable to any adaptive feedforward harmonic control approach, independent of the algorithm used in the adaptation. In the textbook by Widrow and Stearns (1985), the authors highlight the impact of noise on the reference input to an adaptive filter with respect to the optimal Wiener solution. This letter further considers the impact of noise with respect to the sample rate and demonstrates that for single harmonic active noise cancellation (ANC), an optimum sample rate exists for minimizing the mean-squared error. In addition, a single-input, single-output control problem is considered in this work, and the impact of the system dynamics on the optimal Wiener filter in the presence of random (zero-mean) noise is detailed. Results from this study indicate that for the control problem, the minimum mean-squared error which can be achieved is relatively independent of the sample rate, which contradicts the results obtained for the ANC problem. This result stems from the fact that the noise on the reference is filtered by the impulse response of the physical plant and thus becomes correlated with respect to previous iterations in time as will be detailed in the following sections.

\section{OPTIMAL CONTROL FILTER DESIGN THEORY}

A schematic diagram of an adaptive filter implemented to control the response of a structure for some chosen error is illustrated in Fig. 1. The transfer function between the control input and the error is designated by $H_{c e}$ while the transfer function between the disturbance and the error is designate $H_{d e}$. The task of the adaptive filter is to generate a control input, $u_{k}$, such that the output of the system, $y_{k}$, will be equal in magnitude and opposite in phase to the output resulting from the disturbance, $d_{k}$, in the ideal scenario. The cost function is $E\left[e_{k}^{2}\right]$; however, the control input is filtered by the physical system in this case before being summed with the response due to the disturbance (by superposition in a mathematical sense).

In vector form, the mean-squared error can be expressed as follows:

$$
\xi=\mathbf{b}^{T} \mathbf{R b}+\mathbf{W}^{T}[\mathbf{D}+\mathbf{H}] \mathbf{W}+2 \mathbf{W}^{T} \mathbf{C},
$$

where, in words, $\mathbf{R}$ is the input correlation matrix of the reference signal, $\mathbf{b}$ is a vector of the impulse response of the disturbance to error path, $\mathbf{H}$ represents the correlation matrix of the noise signal filtered by the control to error path, D represents the correlation matrix of the filtered reference signal (i.e., filtered-x signal), $\mathbf{W}$ is the vector of adaptive filter coefficients, and $\mathbf{C}$ is the cross-correlation vector between the output of the adaptive filter, $y_{k}$, and the desired response at the error, $d_{k}$.

The optimal solution is obtained by taking the derivative of the mean-squared error with respect to the weight vector and setting the vector equations equal to zero:

$$
\frac{\partial \xi}{\partial \mathbf{W}}=2[\mathbf{D}+\mathbf{H}] \mathbf{W}+2 \mathbf{C}=\mathbf{0} .
$$

Solving for the optimal solution, $\mathbf{W}_{\mathrm{opt}}$, one obtains 


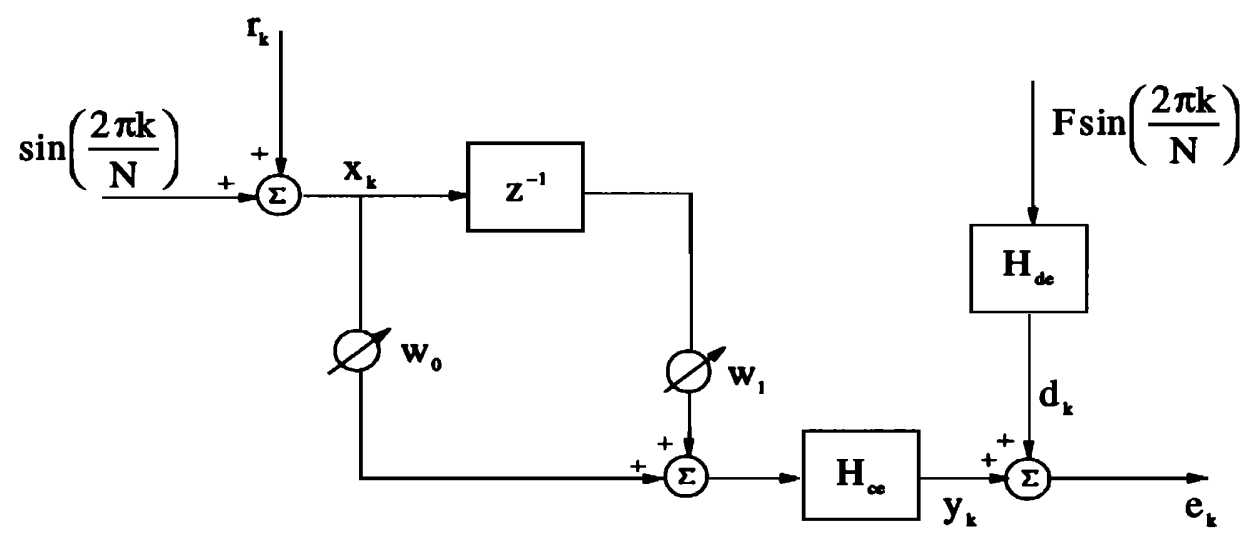

FIG. 1. Schematic diagram of filter structure for control.

$$
\mathbf{W}_{\mathrm{opt}}=-[\mathbf{D}+\mathbf{H}]^{-1} \mathbf{C} .
$$

In the absence of noise, $\mathbf{H}=\mathbf{0}$, and the expression for the unbiased weight vector is obtained. The minimum meansquared error is also expressed in matrix form as follows:

$$
\xi_{\min }=\mathbf{b}^{T} \mathbf{R b}-\mathbf{C}^{T}[\mathbf{D}+\mathbf{H}]^{-1} \mathbf{C} .
$$

As indicated in the previous equation, the minimum meansquared error is controlled by the inverse of $[\mathbf{D}+\mathbf{H}]$, which is composed of the harmonic reference filtered by the physical systems as well as that of the noise filtered by the system, respectively. The minimum mean-squared error differs from that obtained for the optimal filter design in ANC since the noise term is no longer a diagonal matrix. This observation results from the fact that stochastic noise is correlated by the impulse response of a reverberant system, and as will be demonstrated in the results, this off-diagonal contribution will essentially serve to render the adaptive filter implementation independent of the chosen sampling rate with respect to noise on the reference.

\section{RESULTS FROM OPTIMAL FILTER DESIGN}

\section{A. Noise and the optimal filter design for system identification}

To provide an example, the adaptive linear combiner presented in Fig. 6.2 of Adaptive Signal Processing (Widrow and Stearns, 1985) was adopted in this work. For this single frequency ANC problem, the minimum mean-squared error resulting from the optimal filter design with random noise present on the input was computed and is presented in Fig. 2. The variance of the noise is selected such that the signal to noise ratio is $60 \mathrm{~dB}$. As illustrated in Fig. 2, the minimum mean-squared error is achieved at a sample rate between 3 and 6 samples per period. The optimal sampling rate corresponds to $N=4$ for the two coefficient filter and single frequency disturbance. As the sampling rate approaches the $\mathrm{Ny}$ quist frequency, the minimum mean-squared error increases as is the case when the sampling rate is increased beyond that of the optimal. For $N=4$, the input correlation matrix is

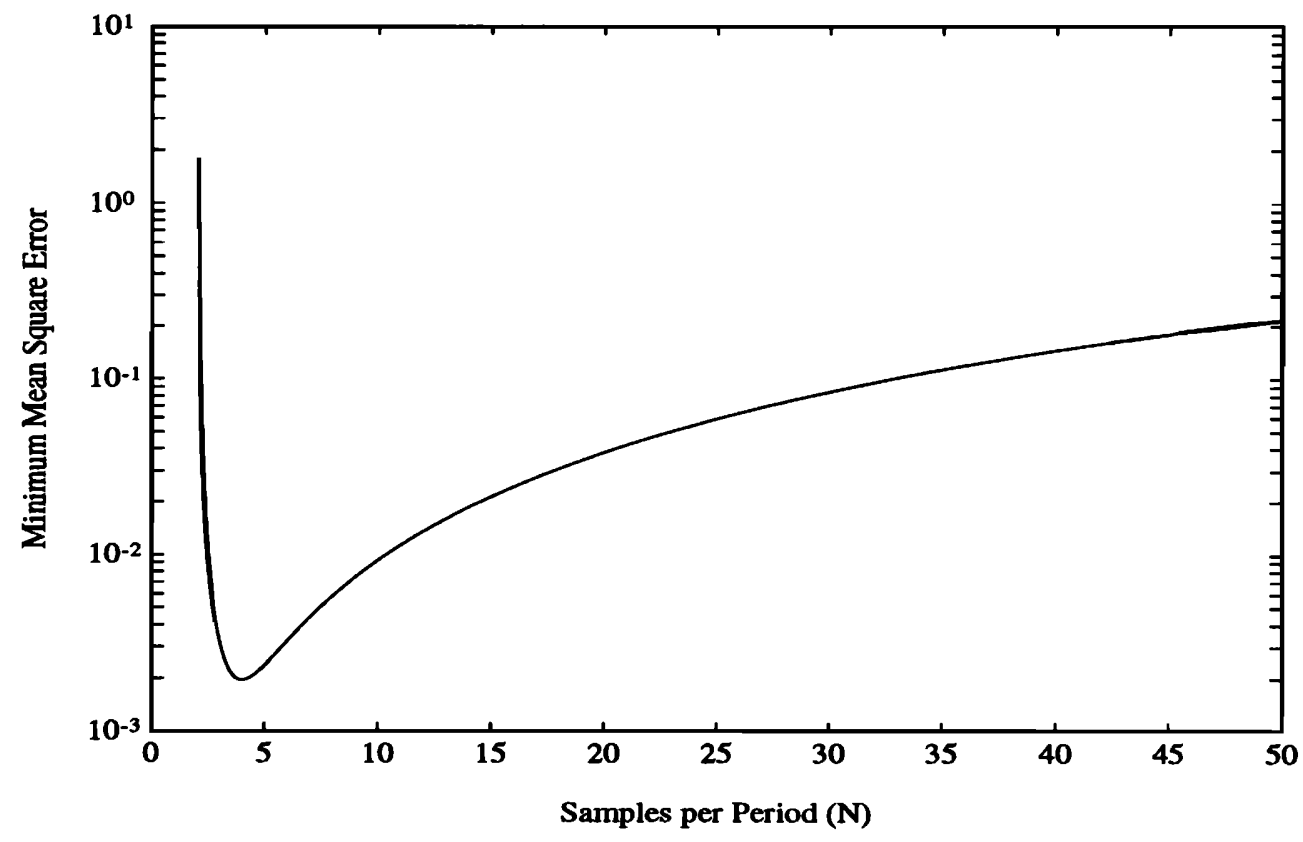

FIG. 2. Minimum mean-squared error as a function of samples per period (harmonic system identification case). 


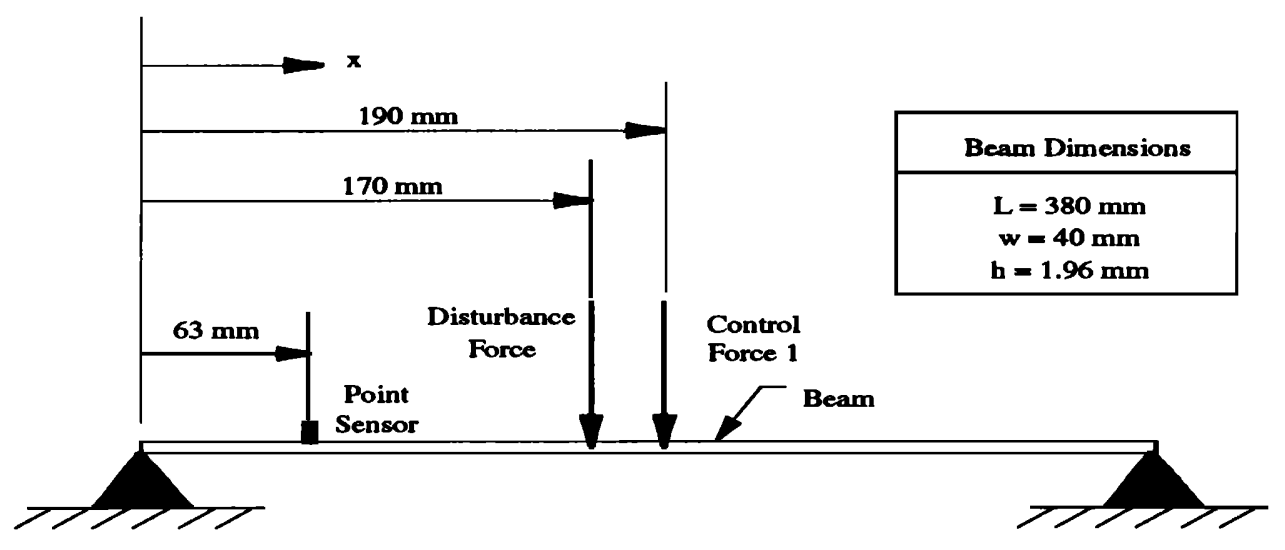

FIG. 3. Schematic diagram of simply supported beam.

diagonal as is the contribution of the noise. Hence the optimal filter can be expressed in terms of a first-order perturbation of the input correlation matrix:

$$
\mathbf{R}=\mathbf{R}_{0}+\mathbf{R}_{1},
$$

where $\mathbf{R}_{0}$ is the input correlation matrix in the absence of noise and $\mathbf{R}_{1}$ is the contribution due to the noise. For $N=4$,

$$
\mathbf{R}_{1}=(\phi / 0.5) \mathbf{R}_{0}=2 \phi \mathbf{I} \text {. }
$$

where $\phi=E\left[r_{x}^{2}\right]$.

Thus the optimal filter design for this case is simply

$$
\mathbf{W}_{\mathrm{opt}}=\mathbf{R}^{-1} \mathbf{P}=\frac{1}{1+2 \phi} \mathbf{R}_{0}^{-1} \mathbf{P}=\frac{1}{1+2 \phi} \mathbf{P},
$$

where $\mathbf{P}$ is the cross-correlation vector between the input reference and the desired response. One can readily show that the minimum mean-squared error can be computed as follows (Widrow and Stearns, 1985; Haykin, 1991):

$$
\xi_{\min }=E\left[d_{k}^{2}\right]-\mathbf{P}^{T} \mathbf{W}_{\mathrm{opt}},
$$

Substituting Eq. (7) into Eq. (8) one obtains an expression for the minimum mean-squared error for $N=4$ :

$$
\xi_{\min }=E\left[d_{k}^{2}\right]-[1 /(1+2 \phi)] \mathbf{P}^{T} \mathbf{P} .
$$

Thus any contribution of noise on the reference will serve to reduce the second term in Eq. (9) and increase the minimum mean-squared error. However, if $\phi$ is small, the performance degradation is reasonably small as well when $N=4$.

In contrast, if $N \neq 4$, the symmetric off-diagonal terms are present and as $N$ approaches infinity, the value of the off-diagonal terms approach that of the diagonal terms, increasing the dependence of the optimal filter design upon the noise contribution. This can be seen upon considering the determinant of $\mathbf{R}$ ( $\mathbf{R}$ can be found in Adaptive Signal Processing, Eq. 6.13, Widrow and Stearns, 1985):

$$
\begin{aligned}
|\mathbf{R}| & =0.5\left[(1+2 \phi)^{2}-\cos ^{2}(2 \pi / N)\right] \\
& =0.5\left[1+4 \phi+4 \phi^{2}-\cos ^{2}(2 \pi / N)\right] .
\end{aligned}
$$

As $N$ approaches 2, the determinant becomes proportional to $\phi$ since $\cos ^{2}(2 \pi / N)$ approaches one, and thus the optimal weights diverge from the solution as a function of $1 / \phi$. Similarly, as $N$ becomes increasingly large, $\cos ^{2}(2 \pi / N)$ again approaches one and the determinant is proportional to $\phi$.
Thus it is obvious as to why the optimal sampling rate occurs at $N=4$ for the two coefficient FIR filter: the noise contribution is insignificant (as long as the signal-to-noise ratio is large) in the determinant of the input correlation matrix and the corresponding contribution to the minimum meansquared error.

\section{B. Noise and the optimal filter design for control}

Fortunately, in the design of an adaptive filter for control applications, the sampling rate does not play an important role with respect to random noise contributions on the input reference. The difference can be attributed to the impulse response of the system (i.e., the filtering of the reference signal through the physical plant). For system identification through ANC, the off-diagonal contribution of the (zeromean) random noise is always zero, owing to the fact that random noise is uncorrelated in time. However, as indicated in Sec. I, an off-diagonal contribution from the noise on the reference input for a control application results from filtering the random noise contribution with the impulse response of the system. Thus the random noise becomes correlated in time, owing to the resonant characteristics of the system.

Given this observation, the off-diagonal contribution of the noise as a function of the sampling rate increases proportionally with respect to the off-diagonal contribution due to the harmonic reference as $N$ is increased for a fixed disturbance frequency. Hence for all values of $N$, the "filtered" input correlation matrix is perturbed proportionally. With a simple first-order perturbation of a real symmetric matrix one can show that if

$$
\mathbf{D}+\mathbf{H} \approx \mathbf{D}+\phi \kappa \mathbf{D},
$$

where $\kappa$ is some constant of proportionality and $\phi$ is the variance of the noise, then

$$
[\mathbf{D}+\mathbf{H}]^{-1} \approx[1 /(1+\phi \kappa)] \mathbf{D}^{-1} .
$$

Since the minimum mean-squared error varies as a function of Eq. (12), as indicated in Eq. (4), any contribution resulting from noise will simply increase the minimum mean-squared error by $\phi \kappa /(1+\phi \kappa)$. Thus for small $\phi$ the effect of the noise is negligible.

An example is provided to demonstrate this observation. For the purpose of this example, a simply supported beam 


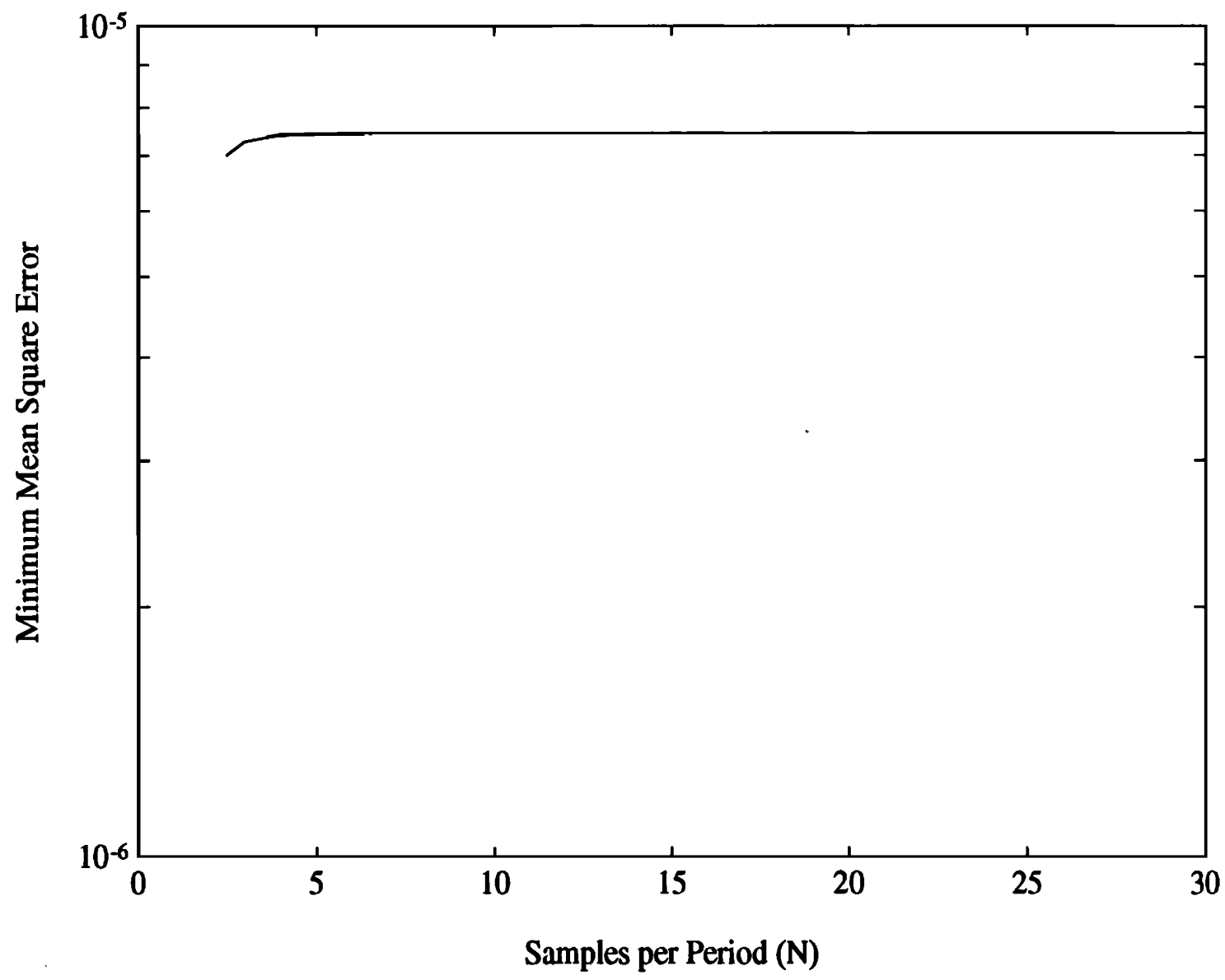

FIG. 4. Minimum mean-squared error as a function of samples per period (harmonic control case).

was selected as the test structure with a single point force disturbance input and a single point force control input. Details of the discrete-time model are omitted for brevity; however, a schematic diagram of the structure is illustrated in Fig. 3. Material properties of the structure were based upon that of steel. The driving frequency was selected at $50 \mathrm{~Hz}$, which is between the first and second natural frequencies of the structure, 31.1 and $124.4 \mathrm{~Hz}$, respectively, for the chosen dimensions and material properties. As indicated in Fig. 4, for random noise with a fixed variance, the minimum meansquared error is relatively independent of the chosen sampling rate in the presence of noise.

\section{CONCLUSIONS}

For single frequency control applications with a two coefficient adaptive FIR filter, if the reference input is contaminated with (zero-mean) random noise, then the optimal sampling rate is 4 samples per period of the harmonic reference. Specifically, if system identification is required as part of the control implementation (as is the case in the filtered-x LMS algorithm), the best estimate of the system response is obtained when the effects of the random noise on the reference are minimized, which occurs when the sampling rate is set at 4 samples per period. In terms of the control implementation of an adaptive FIR filter, this is not the case. Since the noise contribution on the input reference is filtered by the physical system being controlled, the impact of the random noise with respect to the sampling rate is minimal as was indicated in the results. Hence, in terms of the control filter, the sampling rate is essentially arbitrary with respect to noise on the reference.

An alternative method of compensating for the effects of noise on the reference is to increase the length of the FIR filter used in the system identification process (Cowan and Grant, 1985), which ultimately results in greater computational time and effort. If the objective is to maximize the number of control channels which can be utilized for a given digital signal processing rate, one must minimize the computational time required to implement the control. Minimizing the filter length is one method of accomplishing this goal.

Cowan, C. F. N., and Grant, P. M. (1985). Adaptive Filters (Prentice-Hall, Englewood Cliffs, NJ).

Elliott, S. J., Strothers, I. M., and Nelson, P. A. (1987). "A Multiple Error LMS Algorithm and Its Application to the Active Control of Sound and Vibration," IEEE Trans. Acoust. Speech Signal Process. ASSP-35(1), 1423-1434.

Haykin, S. (1991). Adaptive Filter Theory (Prentice-Hall, Englewood Cliffs, NJ), pp. 169-171.

Widrow, B., and Stearns, S. D. (1985). Adaptive Signal Processing (Prentice-Hall, Englewood Cliffs, NJ). 